\title{
Beneficial Effect of Erythropoietin Short Peptide on Acute Traumatic Brain Injury
}

\author{
Bo Wang ${ }^{1}$ Mitchell Kang ${ }^{1} \cdot$ Michelle Marchese $^{1} \cdot$ Esther Rodriguez $^{1} \cdot$ Wei Lu ${ }^{1}$. \\ Xintong $\mathrm{Li}^{1}$ - Yasuhiro Maeda ${ }^{1,2}$ Peter Dowling ${ }^{1,2}$
}

Published online: 29 December 2015

(C) The American Society for Experimental NeuroTherapeutics, Inc. (outside the U.S.) 2015

\begin{abstract}
There is currently no effective medical treatment for traumatic brain injury (TBI). Beyond the immediate physical damage caused by the initial impact, additional damage evolves due to the inflammatory response that follows brain injury. Here we show that therapy with JM4, a low molecular weight 19-amino acid nonhematopoietic erythropoietin (EPO) peptidyl fragment, containing amino acids $28-46$ derived from the first loop of EPO, markedly reduces acute brain injury. Mice underwent controlled cortical injury and received either whole molecule EPO, JM4, or sham-treatment with phosphate-buffered saline. Animals treated with JM4 peptide exhibited a large decrease in number of dead neural cells and a marked reduction in lesion size at both 3 and 8 days postinjury. Therapy with JM4 also led to improved functional recovery and we observed a treatment window for JM4 peptide that remained open for at least $9 \mathrm{~h}$ postinjury. The fulllength EPO molecule was divided into a series of 6 contiguous peptide segments; the JM4-containing segment and the adjoining downstream region contained the bulk of the death attenuating effects seen with intact EPO molecule following TBI. These findings indicate that the JM4 molecule substantially blocks cell death and brain injury following acute brain trauma and, as such, presents an excellent opportunity to
\end{abstract}

Electronic supplementary material The online version of this article (doi:10.1007/s13311-015-0418-y) contains supplementary material, which is available to authorized users.

Peter Dowling

dowlinpc@njms.rutgers.edu

1 Neurology Service, VA Medical Center of East Orange, East Orange, NJ, USA

2 Department of Neurology and Neurosciences, Rutgers New Jersey Medical School, Newark, NJ, USA explore the therapeutic potential of a small-peptide EPO derivative in the medical treatment of TBI.

Keywords Traumatic brain injury $\cdot$ Erythropoietin $\cdot$ Small peptide therapy $\cdot$ Neuroprotection

\section{Introduction}

Traumatic brain injury (TBI) affects up to 10 million people worldwide each year [1]. The health effects of TBI can be debilitating and may result in long-term disability, yet there is currently no effective therapy for TBI. Thus, there remains a pressing need for the development of effective therapeutic agents to combat this condition.

The intact erythropoietin (EPO) molecule is a 165 amino glycoprotein cytokine that was initially identified as a hematopoietic growth factor and has been used extensively for anemia treatment in humans. Whole EPO has received considerable attention over the last decade, owing to reports of its possible neuroprotective and neuroregenerative properties following central nervous system (CNS) injury, including middle cerebral artery occlusion, focal ischemia, subarachnoid hemorrhage, and TBI [2-6]. In several studies, EPO demonstrated efficacy in animal models of stroke and TBI [7-10]. For example, Wang et al. [9] found that EPO aided in angiogenesis and the recovery of neurologic function following stroke injury in a rat animal model, and Brines et al. [5] demonstrated that EPO treatment markedly reduced infarct volume in both ischemic and cortically concussed rat brain. These studies have provided evidence to support the promise of EPO as a beneficial treatment in an array of CNS disorders.

However, the side effect profile of whole-molecule EPO remains a clear disadvantage when administered for any extended period of time. A German clinical trial using 
recombinant human EPO (rhEPO) as a treatment for stroke reported beneficial results post hoc in certain subgroups of patients, despite an unacceptably high mortality in patients treated with EPO [11]. Other studies have found that as EPO elevates hematocrit, side effects such as thrombosis and cardiovascular complications can arise $[12,13]$. Harnessing the neuroprotective capabilities of rhEPO without unacceptable side effects brought about by its hematopoietic effects could therefore lead to the development of an effective TBI treatment strategy.

In this study we designed a novel small, cyclical, EPOderived peptide based on a nonhematopoietic domain within the early sequence [amino acids (AA) 28-46] of the fulllength EPO molecule that we have named JM4. We evaluated its neuroprotective capabilities on a controlled cortical impact (CCI) model of TBI in mice. Groups of injured mice were sham-treated with phosphate-buffered saline (PBS), wholemolecule EPO, or the small EPO-derived peptide JM4 shortly after impact. Neural cell death in the injured brain lesion was quantified by terminal deoxynucleotideyl transferasemediated dUTP nick end labeling (TUNEL) staining at 3 or 8 days following injury. In addition, we determined the therapeutic window for treatment following brain injury by delaying the initial postinjury dosage of JM4. The findings indicate that JM4 provided remarkable CNS protection from cell death and enhanced clinical recovery following acute TBI.

\section{Materials and Methods}

\section{EPO and Peptides}

Whole EPO molecule (Epoetin Alfa; Ortho Biotech Products, L.P., Raritan, NJ, USA) in $2000 \mathrm{U} / \mathrm{ml}$ vial stock was stored at $4{ }^{\circ} \mathrm{C}$. EPO-derived small peptide JM4 (N28GCAEHCSLNENITVPDTKV-46C) was synthesized and purified by United Biochemical Research (Seattle, WA, USA). Peptides were synthesized using solid phase techniques and purified by high-performance liquid chromatography to $90 \%$ purity. The identity and cyclic nature of JM4 were established by matrix-assisted laser desorption/ionization mass spectrometry. JM4 was dissolved in PBS (at $1 \mathrm{mg} / \mathrm{ml}$ ) and kept in small aliquots at $-20{ }^{\circ} \mathrm{C}$ until use.

\section{Animals}

Male C57BL/6 (8-10 weeks old) mice weighing 20-25 g were purchased from Charles River Laboratories (Wilmington, MA, USA) and maintained in a conventional facility receiving standard diet and water ad libitum. The studies were conducted in accordance with the Animal Component of Research Protocol guidelines at the VA Medical Center, East
Orange, NJ, USA, and with the United States Public Health Service's Policy on Humane Care and Use of Laboratory Animals.

\section{Animal Model}

C57BL/6 mice were anesthetized with isoflurane/oxygen inhalation (1.5-2.0\% isoflurane inhalation mixed in $21 \%$ oxygen) and placed in a stereotaxic frame with continuous anesthetic maintenance. Upon establishing the head position in the horizontal plane, a 4-mm craniotomy with the dura left intact over the cortex was produced lateral to the sagittal suture midway between lambda and bregma. A cortical contusion injury was produced utilizing a pneumatically driven 2.5$\mathrm{mm}$ diameter rod tip at $3.5 \mathrm{~m} / \mathrm{s}$ to a depth of $1 \mathrm{~mm}$ with a dwell time of $400 \mathrm{~ms}$ (TBI-0300 Impactor; Precision System Instruments, Lexington, KY, USA). After injury, a 5-mm disk constructed from dental cement was placed over the craniotomy site and adhered to the skull using cyanocrylate. During the early postoperative phase, the animals' temperature was maintained by placing the animals on heating pads. Shamoperated mice received a craniotomy but no cortical impact and the skull was also sealed with a dental cement plate. Mice were assigned randomly to treatment groups postsurgery.

\section{Administration of EPO and JM4 Peptide}

Dose selection of JM4 was determined based on our previous dose-response studies. Logarithmic doses $<10 \mu \mathrm{g} /$ animal were ineffective, while doses $>10 \mu \mathrm{g} /$ animal failed to add any benefit. Intraperitoneal JM4 peptide $(10 \mu \mathrm{g} /$ animal $)$ was administered 15 min after TBI. For determination of the therapeutic window, treatment was delayed following TBI and the initial injection was given at 3, 9, or $24 \mathrm{~h}$. Mice in the intact whole-molecule EPO group received $5000 \mathrm{U} / \mathrm{kg}$ body weight rhEPO (Epoetin Alpha; AMGEN, Thousand Oaks, CA, USA) at 15 min post-TBI. JM4 peptide and EPO were diluted in $200 \mu \mathrm{l}$ filtered sterile PBS prior to injection. Mice in the PBS and sham-operated groups received $200 \mu$ filtered sterile PBS 15 min post-TBI. All mice received subsequent additional JM4 treatments at 24 and $48 \mathrm{~h}$ postinjury.

\section{Blood-Brain Barrier Permeability of JM4}

Anesthetized mice were injected intravenously (i.v.) through the tail vein with an isotope-labeled compound $\left({ }^{3} \mathrm{H}-\mathrm{JM} 4\right.$ or ${ }^{14} \mathrm{C}$-inulin, or both; $10 \mu \mathrm{Ci}$ for each compound in total $100 \mu \mathrm{lBS}$ ). Five minutes after i.v. injection, we performed transcardiac perfusion with $150 \mathrm{ml}$ heparinized saline to flush out the remaining free compound in the blood vessels. Whole brain was then removed and rinsed briefly in cold saline. Tissue was weighed and transferred to $2 \mathrm{ml}$ of Trisethylenediaminetetraacetic acid buffer ( $\mathrm{pH}$ 7.4). Tissue was 
homogenized at maximum intensity for $1 \mathrm{~min}$ (kept cold on ice) and cooled for $10 \mathrm{~min}$ after. Protein was precipitated with $0.5 \mathrm{ml}$ trichloroacetic acid and centrifuged for $10 \mathrm{~min}$ at $2500 \times g$ at $4{ }^{\circ} \mathrm{C}$. To the pellets, $1 \mathrm{ml}$ of Tris- ethylenediaminetetraacetic acid buffer was added and sonicated for $1 \mathrm{~min}$ at maximum intensity. The suspension was transferred to $5 \mathrm{ml}$ scintillation cocktail before radioactivity was counted on a LS6500 machine (Beckman, Indianapolis, IN, USA). In our experiments, we injected mice with $10 \mu \mathrm{Ci}{ }^{3} \mathrm{H}-\mathrm{JM} 4$ and $10 \mu \mathrm{Ci}$ of ${ }^{14} \mathrm{C}$-inulin as a mixture or injected mice with $10 \mu \mathrm{Ci}{ }^{14} \mathrm{C}$-inulin alone. The radioactivity of ${ }^{3} \mathrm{H}$ and ${ }^{14} \mathrm{C}$ across different channels was concomitantly measured and we compared the ratio of ${ }^{3} \mathrm{H} /{ }^{14} \mathrm{C}$ in different tissues to determine movement across membranes.

\section{Determination of the JM4 Therapeutic Window}

The initial dose of EPO-derived JM4 peptide was withheld for various time periods following brain injury for certain experiments. Following injury, groups of mice received their first dose of either 1) PBS (200 $\mu \mathrm{l}) 15 \mathrm{~min}$ post-TBI; 2) JM4 (10 $\mu \mathrm{g}$ /mouse) $15 \mathrm{~min}$ post-TBI; 3) JM4 (10 $\mu \mathrm{g} /$ mouse) $3 \mathrm{~h}$ post-TBI; 4) JM4 (10 $\mu$ g/mouse) $9 \mathrm{~h}$ post-TBI; or 5) JM4 (10 $\mu \mathrm{g} /$ mouse) $24 \mathrm{~h}$ post-TBI. All mice received subsequent treatment doses at $24 \mathrm{~h}$ and $48 \mathrm{~h}$ following injury. Mice in the 24-h delayed treatment group received a total of 2 doses rather than 3 doses of treatment. Mice were sacrificed 3 days postTBI.

\section{In situ TUNEL Detection and Cell Death Quantification}

Mice were sacrificed at 3 or 8 days postinjury and brains were collected and frozen immediately on dry ice for cryosectioning. Frozen brains were serially sectioned in coronal cuts onto 15 slides with 12 sections $(16 \mu \mathrm{m})$ per slide extending from the anterior to the posterior edge of the lesion. Sections were placed so that each individual slide contained a representative sampling of the entire lesion within the injured hemisphere.

Cell death in the mouse brains was detected using a modification of the Apoptag (Millipore, Billerica, MA, USA) TUNEL method. Serial $16-\mu \mathrm{m}$ cryosections were mounted and acetone-fixed for $10 \mathrm{~min}$. The slides were rehydrated and postfixed in 2:1 ethanol:acetic acid for $11 \mathrm{~min}$ at $20{ }^{\circ} \mathrm{C}$. The slides were washed thoroughly after postfixation, incubated with equilibration buffer for $10 \mathrm{~min}$, then immediately reacted with working strength TdT enzyme for $60 \mathrm{~min}$ at $37{ }^{\circ} \mathrm{C}$. The TUNEL reaction was detected using Cy 3 antidigoxin (1:200; Jackson ImmunoResearch, West Grove, PA, USA) staining for $45 \mathrm{~min}$.

Images were captured using a fluorescent Olympus (Center Valley, PA, USA) BX60 microscope fitted with a Retiga (Surrey, BC, Canada) 2000R digital camera at $10 \times$. The total number of TUNEL-positive cells in lesioned hemisphere cryosections was quantified using digital imaging software IP Lab 4.0 (BD Biosciences, Franklin Lakes, NJ, USA) and labeled cells were quantified by two independent observers blinded to animal treatment groups. Four slides of injured brain (slides 1, 5, 10, and 15) were quantified so that the 48 sections were representative of the entire lesion. Counting was accomplished by the computer software with adjustment of parameters on Q-imaging (Surrey, BC, Canada) quantification software, to account for the intensity of the TUNEL stain.

The injury area containing TUNEL-positive cells was determined at $10 \times$ magnification using the same images employed for the quantification of TUNEL-positive cells. The area containing the positive cells was outlined by hand. All 12 cryostat sections on an individual slide were considered in determining the quantification of area. Quantification was performed using IP Lab 4.0 software.

\section{Assessment of 6 Contiguous Fragments of Whole EPO Peptide to Isolate the TBI Cell Death Blocking Effect}

Six peptide fragments comprising nearly the entire AA sequence of whole EPO were synthesized (United Biochemical Research, Seattle, WA, USA). The peptide fragments, termed EP-P\#1-EP-P\#6, started at the N-terminal side of EPO and each spanned $25 \mathrm{AA}$, so that EP-P\#5 ended at AA residue 125. EP-P\#6 contained AA residues D136-166R. The experimental design was the same as the CCI protocol, including the cortical impact, administration of peptide (see "Administration of JM4" section), TUNEL detection, and quantification.

\section{Simple Neuroassessment of Asymmetric Impairment Score}

Animals received CCI and were treated with PBS $(n=6)$ or JM4 $(n=6)$ or received sham surgeries $(n=6)$ in which a craniotomy was performed with skull capping afterward but no CCI. TBI animals were assessed for clinical recovery on days $1-8$ and on day 10 following TBI by 2 blinded independent evaluators. A modified version of the simple neuroassessment of asymmetric impairment (SNAP) test was used [14]. This version excluded the visual field test and combined the gait/ posture portion with the head tilt. The baton test was separated into 2 categories, speed and accuracy, with a maximum of 2 points each. SNAP scores of 0 indicated no deficit, and the total possible score (except baton) for each test was 5 . The specifics of scoring are described in the Supplementary Table S1.

\section{Statistical Analysis}

The data (mean \pm SEM) between groups were compared by 1 way analysis of variance (ANOVA) tests, followed by Tukey's 
test for post hoc comparison of individual group means between PBS-, rhEPO-, and JM4 peptide-treated animals. The analysis of composite SNAP clinical scoring utilized a mixed design study ANOVA with Tukey's post hoc test using a within-subject factor of time and a between-subject factor of treatment given (PBS, JM4, or whole EPO). A value of $p<0.05$ was considered statistically significant. Figures include all individual data points, with each point representing the data collected from an experimental animal. Rectangular boxes around the data points were added for visual enhancement of the data ranges. Data analyses were performed with Prism 5.0 software (GraphPad, La Jolla, CA, USA).

\section{Results}

\section{EPO-derived JM4 Peptide Crosses the Blood-Brain Barrier to Block Cell Death in TBI}

We determined that radiolabeled 3H-JM4 does, indeed, pass the blood-brain barrier. Simultaneous i.v. injections of $3 \mathrm{H}-$ JM4 and 14C-Inulin were performed in normal mice, followed by serum collection at $5 \mathrm{~min}$ postinjection and then a careful washout protocol and collection of the brain. Nonpermeable 14C-Inulin served as a negative control. Data indicated that the brain/serum ratio of JM4 greatly exceeds that of inulin, indicating JM4 likely crosses the blood-brain barrier (Table 1).

To evaluate the effect of JM4 treatment on acute TBI after cortical contusion, mice were either sham treated with PBS, treated with JM4, or treated with whole EPO molecule 15 min after injury. Mice were treated for 3 days and sacrificed at $72 \mathrm{~h}$, while others were sacrificed later at 8 days postinjury. The frozen brain tissue was serially sectioned and stained using the TUNEL assay to identify cells with nuclear DNA fragmentation, indicative of cell death. The stained tissues were visualized using fluorescent microscopy, and TUNELpositive cells were quantified.

TUNEL staining revealed a drastic reduction in TUNELpositive cells in the injured hemisphere of animals treated with JM4 compared with PBS-treated control animals (Fig. 1A, B).

Table 1 The brain/serum ratio of JM4 greatly exceeds that of inulin, indicating that JM4 likely crosses the blood-brain barrier

\begin{tabular}{llll}
\hline Brain/serum ratio & Normal \#1 & Normal \#2 & Normal \#3 \\
\hline${ }^{3} \mathrm{H}-J \mathrm{M} 4$ & 2.55 & 2.71 & 2.41 \\
${ }^{14} \mathrm{C}-$-Inulin & 0.35 & 0.99 & 1.22 \\
\hline
\end{tabular}

The influx rate was $0.34 \pm 0.06 \mu \mathrm{l} / \mathrm{g} / \mathrm{min}$, which is the typical value expected for a peptide the size of JM4 $(\sim 2 \mathrm{kD})$ crossing the blood-brain barrier by transcellular diffusion
Animals treated with whole-molecule EPO served as a positive control and also exhibited sharply reduced numbers of TUNEL-positive cells. Additionally, TUNEL-stained JM4treated brains tended toward a restricted pattern of cell death confined to the immediate area of the lesion core, while PBS control-treated tissue displayed prominent, widespread expansion of cell death outward from the core, often extending into the basal ganglia and hippocampus (Fig. 1A).

\section{Cell Death Quantification Following Brain Injury}

To quantify the amount of cell death within the injury area of each animal, brains were serially cryosectioned from anterior to posterior. The slides were visualized by fluorescent $\mathrm{Cy} 3$ detection and quantified. The injury zone in the lesioned hemisphere was quantified by following the TUNELnegative margin immediately outside of the TUNEL-reactive zone. The contralateral hemisphere in each brain displayed rare or no TUNEL-positive cells.

Both whole EPO and JM4 were found to reduce significantly the quantity of TUNEL-positive cells within the injured hemisphere of animals receiving therapy for 3 days $(p<0.001$; Fig. 2A). Whole EPO therapy produced a $60 \%$ reduction in cell death compared with animals receiving sham (PBS) treatment $(p<0.001)$. JM4 therapy performed comparably, with a $65 \%$ reduction in the number of TUNEL-positive cells $(p<0.001)$.

This neuroprotective effect was also seen in animals treated for 3 days with sacrifice delayed until 8 days postinjury $(p<0.001$, ANOVA; Fig. 2B). JM4 treatment elicited a $55 \%$ reduction in TUNEL-positive cells $(p<0.05)$. In contrast, TBI animals treated with whole EPO for 3 days and sacrificed at 8 days postinjury displayed a more limited, nonsignificant $30 \%$ reduction $(p=0.091)$. Though cell death at 8 days postinjury was not significantly diminished by whole EPO treatment, a trend towards a positive treatment effect was still present.

To further assess the extent of lesion size within the injured hemisphere, the total area containing TUNEL-positive cells in each injured brain was calculated. To accomplish this, one slide from each animal containing representative sections of the whole brain was TUNEL-stained and the TUNEL-positive area was defined by manually tracing around the TUNELpositive margins of the lesion using IP Lab 4.0.

Treatment with either whole EPO or JM4 resulted in a significant reduction in lesion size compared with PBS sham-treated animals ( $p<0.001$, ANOVA; Fig. 3A). In JM4treated animals sacrificed 3 days postinjury, TUNEL staining revealed a reduction of $>55 \%$ in lesion area compared with sham-treated animal lesions $(p<0.001)$. A $45 \%$ reduction in lesion size was observed in whole-molecule EPO-treated animals $(p<0.01)$. In animals treated for 3 days but sacrificed at 8 days, JM4 continued to induce a significant reduction in 
Fig. 1 Low magnification $(2 \times)$ of cryostat-sectioned brain lesions comparing JM4-treated traumatic brain injured (TBI)-animal to sham-treated TBI control. The top panel shows the damaged areas [hematoxylin and eosin (H\&E) staining]. The middle panel shows fluorescent-labeled nuclear terminal deoxynucleotidyl transferase-mediated dUTP nick end labeling (TUNEL) staining for DNA fragmentation (TUNEL method with $\mathrm{Cy} 3$ detection). The lower panel shows higher magnifications $(20 \times)$ of TUNELstained lesions. The lesion in the JM4-treated animal shows markedly reduced cell death (TUNEL stain) and size compared with the phosphatebuffered saline (PBS)-treated control lesion at 3 days postinjury.

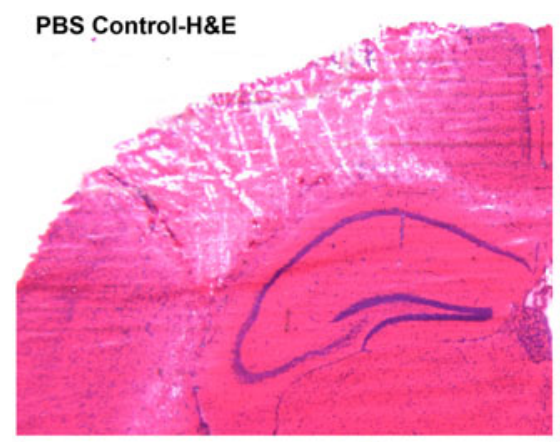

JM4-H\&E
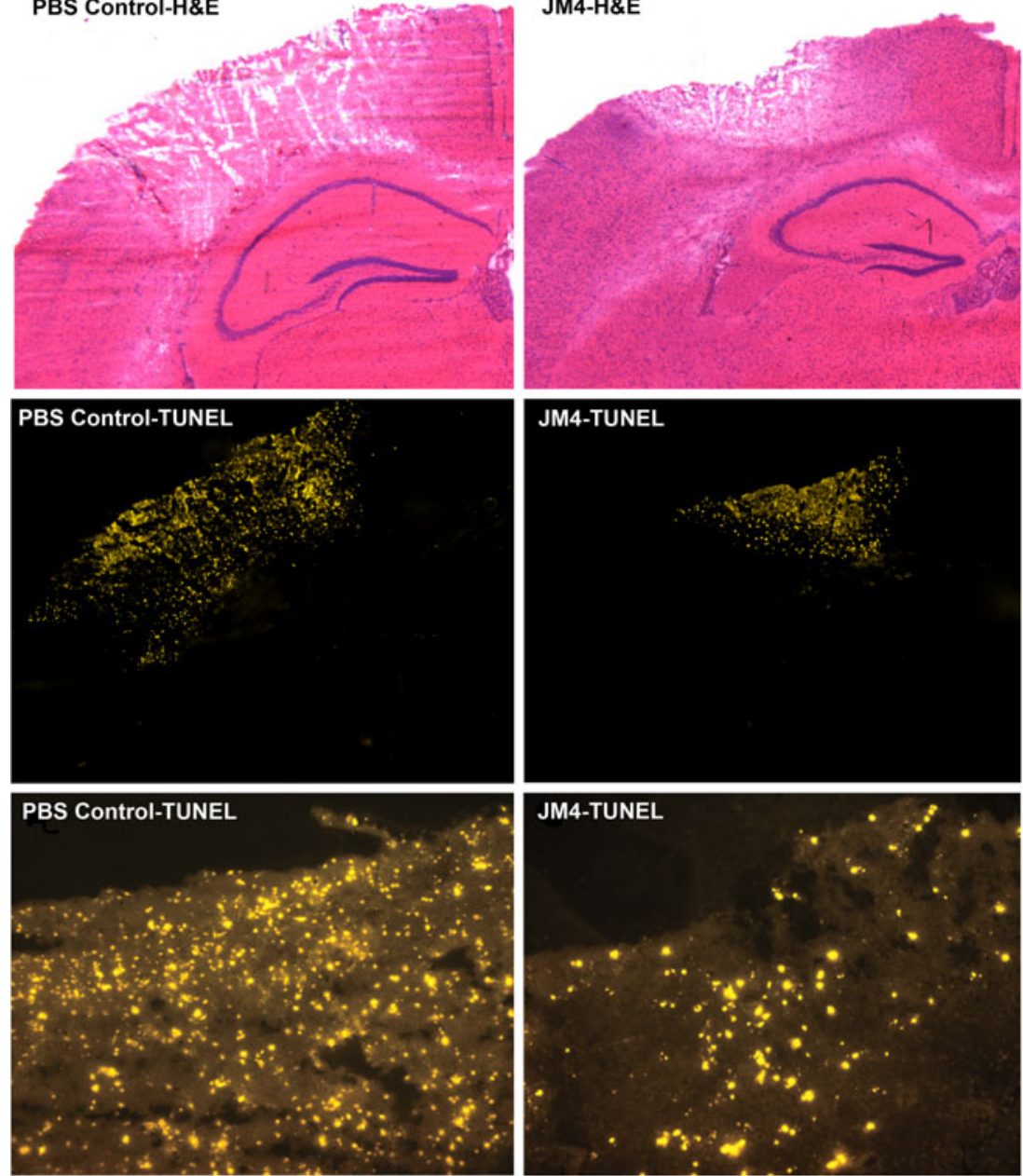

lesion size compared with sham PBS-treated controls $(p<0.05$; Fig. 3B). However, animals treated with wholemolecule EPO and sacrificed 8 days post-TBI failed to demonstrate a significant reduction in lesion area $(p=0.07)$. As with total dead cell count, whole EPO treatment induced a positive, though not significant, effect on lesion area reduction.

\section{Clinical Assessment of Impairment}

We tried several strategies designed to quantify neurological deficit consistently, without success, in the early stages of our study. However, Shelton et al. [14] introduced the SNAP test, which we found quite useful in reproducibly quantifying neurological impairment in brain-injured mice when tested by blinded observers. To assess each animal's degree of clinical impairment, we developed a modified version of the SNAP test (Supplementary Table S1). Animals were followed and assessed by blinded observers on days 1-8 and on day 10 post-TBI (Fig. 4). The data were assessed using a mixeddesign ANOVA and indicated a main effect on days postTBI $[\mathrm{F}(8,120)=35.94, p<0.001]$ and a main effect on treatment $[\mathrm{F}(2,15)=507.94, p<0.001]$, as well as days post$\mathrm{TBI} \times$ treatment interaction $[\mathrm{F}(16,120)=15.09, p<0.001]$. Tukey's post hoc test indicated that JM4-treated mice performed better than PBS-treated mice in composite clinical scoring $(p<0.05)$, and exhibited significantly less clinical deficit. Mice receiving sham surgeries in which only the craniotomy was performed but no cortical injury took place performed better clinically than either JM4- or PBS-treated mice $(p<0.05)$. The maximum attainable score was 29 , which would indicate complete impairment in every category; however, the highest individual score on any day among all animals throughout the experiment was 13 . Typically, mice ranged from 3 to 10 in scoring. Therefore, absolute reductions in SNAP clinical score ranged from 0 to 4 points on any given day.

\section{Therapeutic Window}

To simulate situations where drug delivery to the patient following TBI would not be immediately feasible, we determined the duration of the therapeutic window during which short peptide JM4 treatment was effective. 

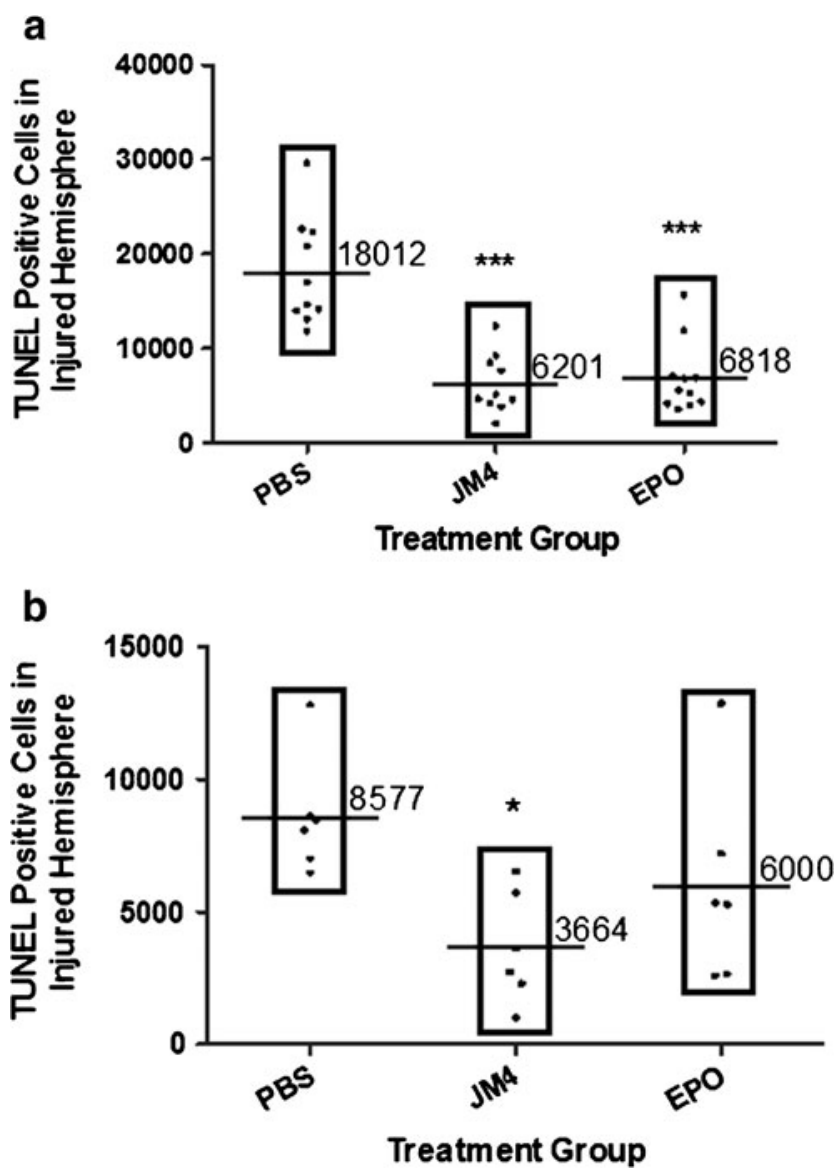

Fig. 2 Whole-molecule erythropoietin (EPO) and its derivative peptide JM4 profoundly reduce cell death in the impacted hemisphere. (A) Treatment with EPO $(n=11)$ or JM4 $(n=10)$ significantly decreased terminal deoxynucleotidyl transferase-mediated dUTP nick end labeling (TUNEL)-positive cells in animals receiving 3 days of treatment compared with phosphate-buffered saline (PBS) sham-treated $(n=10)$ TBI animals $(* * * p<0.001)$. (B) A positive treatment effect was also seen in JM4-treated animals 8 days postinjury $(n=6$ for each group; $* p<0.05)$.

We withheld the initial treatment of JM4 peptide for 3, 9, or $24 \mathrm{~h}$ postinjury. Mice with an initial delay of treatment for 3 or $9 \mathrm{~h}$ subsequently received additional JM4 at 24 and $48 \mathrm{~h}$ postinjury. Mice that received their first dose $24 \mathrm{~h}$ postinjury and a subsequent dose at $48 \mathrm{~h}$ postinjury did not receive a third. Mice were sacrificed at $72 \mathrm{~h}$ postinjury and the effect of treatment delay was determined on the injured brain with TUNEL staining.

TUNEL staining revealed greatly depressed levels of cell death in mice treated 15 min postinjury with the standard 3day JM4 regimen (50\% decrease) compared with shamtreated brain-injured controls ( $p<0.01 ;$ Fig. 5). Furthermore, mice receiving delayed initial treatment with JM4 peptide at $3 \mathrm{~h}$ postinjury showed marked cell death attenuation (50\% decrease) equivalent to mice treated 15 min postinjury $(p<0.01)$. Mice that were administered initial JM4 after a 9-h delay postinjury still demonstrated a slightly greater diminished number of TUNEL-positive cells (65\% decrease) a
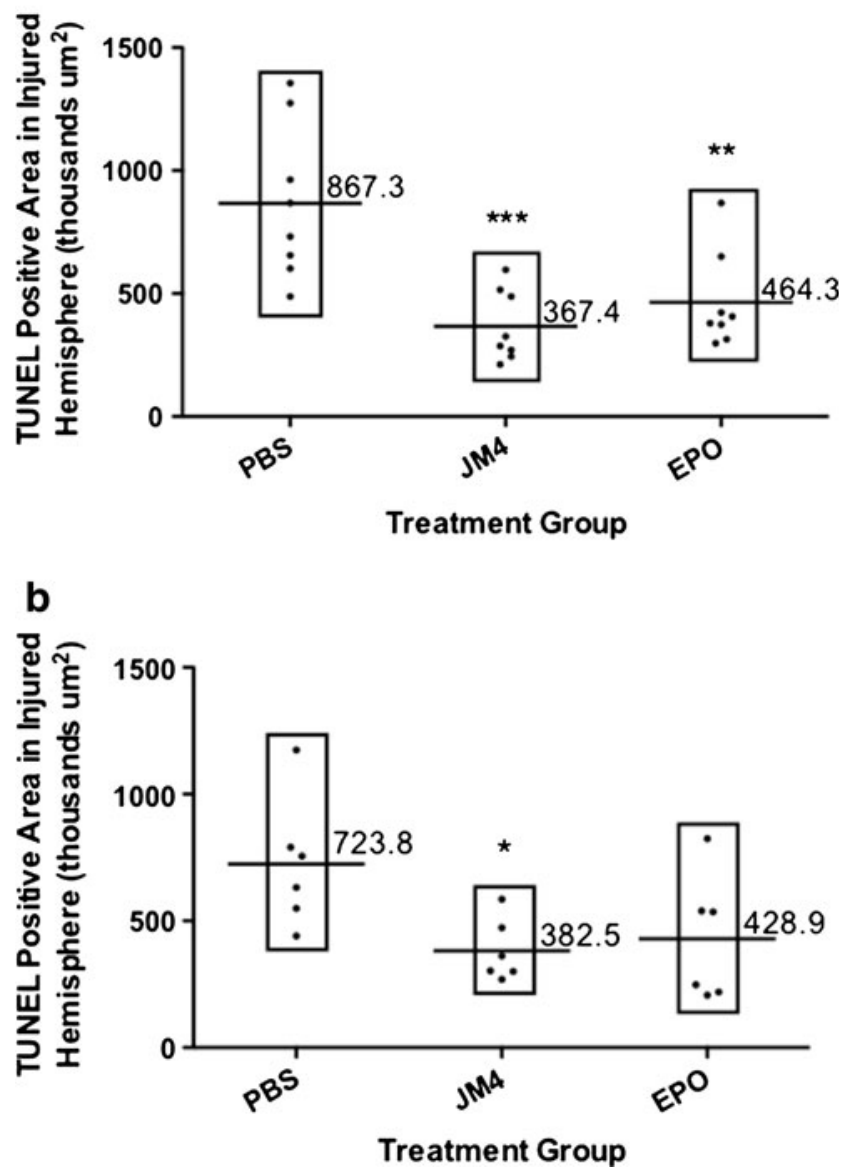

Fig. 3 Effect of JM4 therapy on the lesion area within the injured hemisphere (evaluated at 3 days and 8 days postinjury). For this experiment, a terminal deoxynucleotidyl transferase-mediated dUTP nick end labeling (TUNEL)-labeled slide containing sections spanning the entire hemisphere was used to quantify the total area containing dying cells. The JM4-treated group showed a marked reduction $(50 \%)$ in lesioned area compared with phosphate-buffered saline (PBS) shamtreated animals at both (A) 3 days $(* * p<0.01, * * * p<0.001)$ and (B) 8 days postinjury $(* p<0.05)$. EPO $=$ erythropoietin

compared with sham-treated TBI controls $(p<0.001)$. Mice receiving no treatment until $24 \mathrm{~h}$ postinjury displayed reduced cell death compared with controls $(30 \%)$, but the treatment effect failed to achieve significance. The sample size for the 24-h group was limited to 3 animals.

\section{Neuroprotection is Limited to Early Segments of the Whole EPO Molecule}

Six contiguous peptide fragments spanning the bulk of the entire EPO sequence were synthesized. Each fragment consisted of about $1 / 6$ of the primary structure of wholemolecule EPO, and each fragment was tested for neuroprotection efficacy in our TBI animal model and subsequent cell death attenuation quantified by TUNEL stain. Following injury, mice were treated with either PBS, JM4 peptide, or 1 of 6 


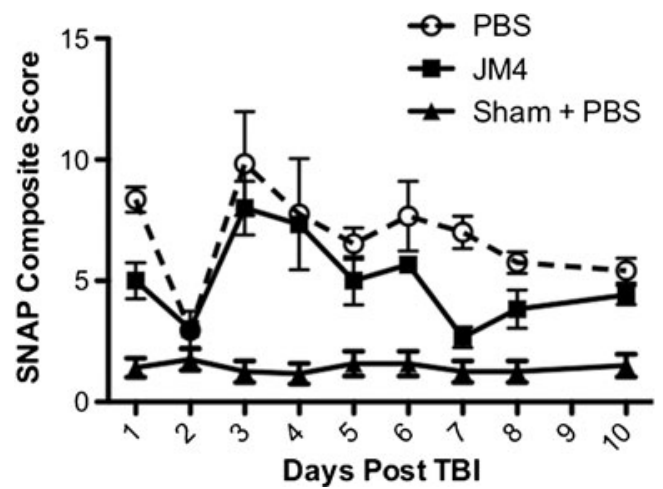

Fig. 4 A modified version of the simple neuroassessment for asymmetric impairment (SNAP) was used to determine if JM4 therapy has a treatment effect on neurologic deficit in brain-injured animals. Evaluation was performed by blinded observers postinjury. JM4-treated traumatic brain injury (TBI) mice showed significant improvement in neurological deficit compared with phosphate-buffered saline (PBS) sham-treated TBI mice on a 29 -point scale (score $0=$ no deficit) following TBI. Significant positive effects were observed in this mixed design study, particularly as time progressed when comparing PBS- and JM4-treated animals $(p<0.05)$. Data are mean $\pm 95 \%$ confidence interval

EPO peptide fragments. TBI animals were treated with an initial dose $15 \mathrm{~min}$ postinjury and treated every $24 \mathrm{~h}$ for a total of 3 doses. Some groups of JM4-treated mice expressed significantly diminished quantities of TUNEL-positive cells $(p<0.005$; Fig. 6), and another short EPO peptide, pyroglutamate helix B surface peptide (pHBSP) [15], an 11 mer peptide comprised of select AA from the hydrophilic face of the B helix of EPO (AA 56-82), also showed a substantial reduction in TUNEL-positive cell numbers $(p<0.01)$. The EPO fragment peptides were labeled EP-P\#1-EP-P\#6, with each increasing number corresponding to a peptide derived from a region closer to the C-terminal of EPO. The complete sequence of JM4 lies within EP-P\#2, and the sequences of helix B surface peptide (HBSP) and pHBSP (small peptide derivatives of EPO) lie partially within EP-P\#3 and EP-P\#4 $[15,16]$. Of these EPO segments, EP-P\#2, which contained the JM4 sequence, demonstrated the most significant reduction in quantity of TUNEL-positive cells within injured brain relative to the controls. The EP-P\#3 segment also induced a reduction in TUNEL-positive cells. Therapy with EP-P\#5 demonstrated an appreciable though more marginal level of reduction in brain following TBI $(p<0.05)$.

\section{Discussion}

The purpose of this study was to investigate the tissueprotective effects of a nonhematopoietic domain derived from whole-molecule EPO on TBI in a mouse animal model. Attenuated cell death in JM4-treated animals appears to correlate with reduced clinical deficit following TBI. We also showed that despite an initial treatment delay, JM4 boasted a potent

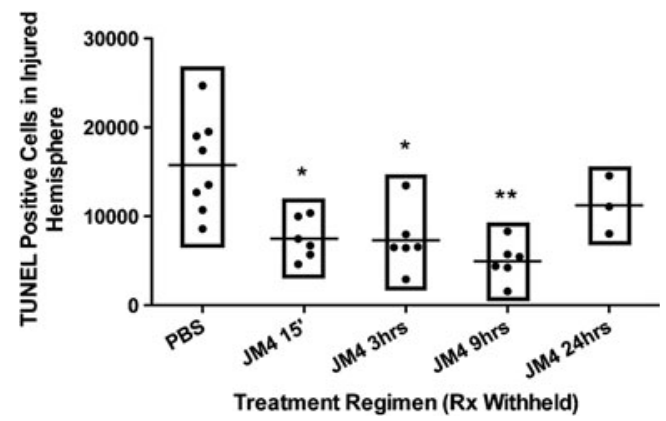

Fig. 5 Therapeutic window for JM4 therapy remains open for at least $9 \mathrm{~h}$ postinjury. Mice were given identical cortical injuries and separated into groups. Phosphate buffered saline (PBS) sham-treated control traumatic brain injury (TBI) animals were injected with $200 \mu \mathrm{l}$ PBS 15 min postTBI and 3 separate groups of TBI animals were administered an initial dose of JM4 (10 $\mu \mathrm{g} /$ mouse) at either $15 \mathrm{~min}, 3 \mathrm{~h}$, or $9 \mathrm{~h}$ postinjury, and subsequently treated once daily for 2 additional days before sacrifice. Cell death was quantified using the terminal deoxynucleotidyl transferasemediated dUTP nick end labeling (TUNEL) reaction. Treatment groups given initial treatment at $15 \mathrm{~min}, 3 \mathrm{~h}$, or $9 \mathrm{~h}$ postinjury all showed major reductions in TUNEL-positive cell number compared with the shamtreated control group. The effectiveness of JM4 therapy, despite delayed initial administration, indicates that the treatment window of JM4 extends for at least $9 \mathrm{~h}$ after TBI $\left({ }^{*} p<0.01,{ }^{* *} p<0.001\right)$.

therapeutic effect that extended for at least the first $9 \mathrm{~h}$ following injury. Finally, the neuroprotective characteristics of whole EPO appear to be contained within the early portion of the intact EPO sequence. The most profound cell death-blocking effects were restricted to the JM4-containing sequence, EP$\mathrm{P} \# 2$, and, to a slightly lesser degree, EP-P\#3.

JM4 peptide represents a newer class of EPO derivative composed of a small peptide region derived from the $\mathrm{AB}$ loop of the whole EPO molecule. We aimed to define a region of the EPO molecule that retained therapeutic efficacy in neuroprotection yet eliminated the undesired hematopoietic interactions that have severely restricted the use of whole EPO treatment in TBI [17]. The JM4 sequence (N28GCAEHCSLNENITVPDTKV-46C) was chosen for the presence of a disulfide bond capable motif comprised of 2 cysteine residues in the first loop of EPO, allowing for a cyclic structure in solution to enhance stability. Other EPO derivatives based on the same desire to eliminate undesired hematopoiesis have been studied for their use in brain injury, including HBSP and a pHBSP (also known as ARA290) [16]. HBSP and its analog pHBSP have been found to be tissue protective in models of stroke [18], cardiovascular disease [19, 20], experimental autoimmune encephalomyelitis (EAE) [21], and TBI [15]. In our investigation, JM4 also significantly limited the size of the lesion in injured brains following TBI (Fig. 3). We elected to measure cell death with TUNEL staining, which has been used extensively to assess for cell death following TBI $[22,23]$. We additionally observed significant attenuation of cell death in brain lesions whether treated with JM4 or EPO, indicating that equivalent levels of neuroprotection can be afforded by both whole-molecule EPO and JM4 (Fig. 2). 
Fig. 6 The majority of the neuroprotection afforded by erythropoietin (EPO) is contained within a select early segment of the whole EPO molecule. (A) Treatment with EP-P\#2 or EP$\mathrm{P} \# 3$ profoundly reduces cell death in the affected hemisphere, significantly decreasing terminal deoxynucleotidyl transferasemediated dUTP nick end labeling (TUNEL)-positive cells in animals receiving 3 days of treatment compared with phosphate-buffered saline (PBS) sham-treated traumatic brain injury (TBI) animals $[n=8$ for each group, $p<0.0001$ (EP-P\#2), $p<0.005$ (EP-P\#3)]. (B) Map of whole EPO sequence, showing the positions of EP-P \#1-6, JM4, and short peptides helix B surface peptide (HBSP)/pyroglutamate helix B surface peptide (pHBSP). The HBSP/pHBSP* in the figure key indicates that the molecules HBSP and pHBSP are not reflected by the primary structure of EPO and are rather derived from the aqueous face of three dimensional structure of helix B a

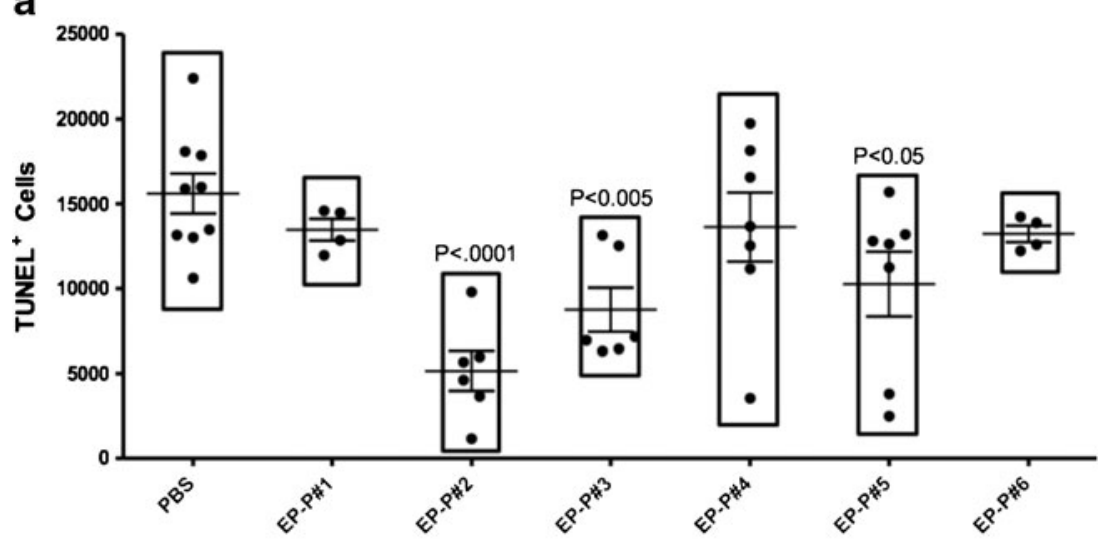

b

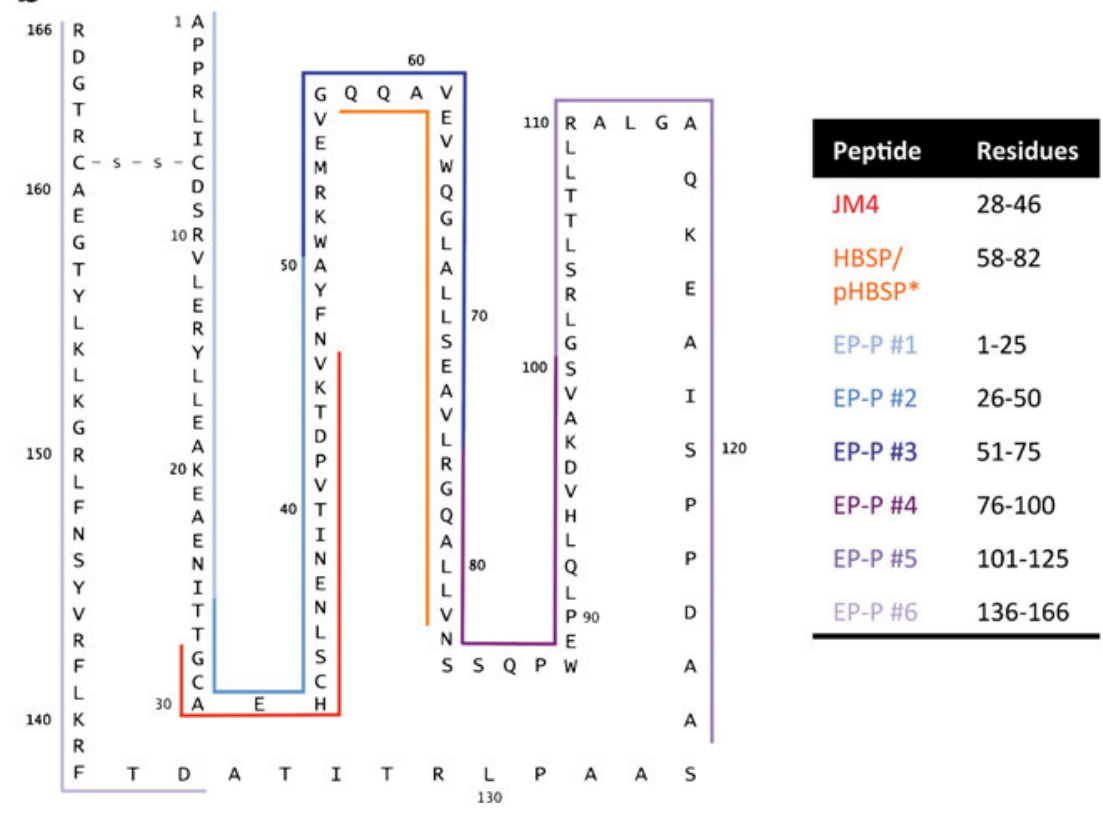

When the whole EPO molecule was divided into consecutive fragments, our data support the notion that only early segments of the molecule contain the significant neuroprotective effects afforded by EPO (Fig. 6A). This neuroprotective region (AA 28-82) contains the sequences for JM4, HBSP, and pHBSP (Fig. 6B), and, of note, the second fragment (EP-P\#2), which contains the JM4 sequence demonstrated to have the most profound cell death-blocking effect. Our data contribute to a small yet steadily growing body of literature demonstrating neuroprotective properties of EPO and the potential for its small peptide derivatives to take on a prominent role in the treatment of brain injury.

Several human trials have revealed that whole-molecule EPO treatment may lead to significantly increased incidence of thrombotic events. More alarmingly, in a phase II/III study on the use of rhEPO for treatment of stroke, the death rate among patients treated with rhEPO was elevated to $16.4 \%$ versus $9.0 \%$ in the placebo group $(p=0.01)$ [11]. These findings affected a recently published clinical study investigating the use of EPO following TBI to such a degree that the initial 3-dose regimen of EPO was decreased midway through the study to only a single initial dose of EPO after injury [24]. The study failed to report significant benefit in neurological recovery in patients, but it is important to note that the dosage used was about $1 / 10$ of the most effective clinical dose in animal TBI models and that more than half of enrolled patients had a Glasgow Coma Score of 8 or less (severely impaired). In contrast, in our animal model most mice were conscious, alert, and moving within 30-60 min of their TBI procedures and received a complete EPO 3-dose equivalent of JM4. From the mortality data we might gather that the concentration of EPO required in circulating plasma to elicit its neuroprotective effects may risk the activation of erythropoiesis and downstream side effects.

In response to the side effects brought on by EPO treatment, derivatives of the whole EPO protein, including our 
compound, JM4, have been designed and studied in animal models [25-27]. The goal of the design was to maintain the tissue-protective capabilities of the EPO molecule while eliminating the erythropoietic characteristics that lead to the previously described complications. JM4 has been previously demonstrated to have no effect on hematocrit in animals [17].

We additionally developed a clinical correlate to follow the level of TBI deficit during the disease course following injury. Brain-injured mice treated with JM4 showed fewer deficits than their PBS sham-treated counterparts during the postTBI course (Fig. 4). Scores for injured mice were significantly higher compared with baseline scores set by sham-operated uninjured mice, allowing us to discount the effects of the craniotomy and other surgical factors unrelated to CCI. From our experience, SNAP provides a useful clinical correlate to track neurologic impairment and recovery [14]. Our results indicate that JM4 remains effective in limiting neurological deficit following injury over a 10-day trial. Interestingly, on day 2 , the composite SNAP scores of the JM4- and PBS-treated groups fell dramatically, only to increase again on day 3. TBI has been associated with delayed activation of CCL2 and CCL20, as well as secondary delayed activation of interleukin (IL)- $1 \beta$ and IL-4 in a rat CCI model, suggesting a temporal relationship between the cytokine profile, their targeted cellular infiltrates, and observed clinical deficits [28]. Exploration of EPO as an immunomodulatory agent has revealed that EPO reduces levels of the proinflammatory cytokines tumor necrosis factor (TNF)- $\alpha$, IL-1 $\beta$, interferon (IFN)- $\gamma$, IL- 6 , and intracellular adhesion molecule 1 following EPO treatment in TBI and EAE [29-31]. Other studies in EAE and cerebral malaria have demonstrated that whole-molecule EPO treatment can modulate a highly unstable autoimmune environment into an immune system of relatively normal activity, limiting peripheral and CNS infiltration by CD4+ and CD8+ T cells, reducing the number of mononuclear cells and dendritic cells, while suppressing $\mathrm{T}$ helper 17 cells, IFN- $\gamma$, and TNF mRNA [29-32]. In some studies EPO also induced elevation of IL10 and FoxP3 + regulatory $\mathrm{T}$ cells, factors that contribute toward immune silencing.

An important and highly practical aspect of the design of novel therapeutics is the time frame in which the molecules maintain a high level of efficacy. Whole-molecule EPO reportedly loses protective effectiveness $6 \mathrm{~h}$ postinjury [5]. An increase in therapeutic window duration by a few hours could represent a significant advantage over existing therapies. Surprisingly, delaying initial treatment of brain-injured mice with JM4 peptide for $9 \mathrm{~h}$ seemed to reduce TUNEL cell death as much as immediate treatment with JM4 (Fig. 5). These findings demonstrate that the therapeutic window for EPOderived JM4 treatment is open for at least $9 \mathrm{~h}$ postinjury and likely extends further toward the 24-h postinjury mark. Studies have observed that TNF- $\alpha$ levels peak between 3 and $8 \mathrm{~h}$ following TBI $[33,34]$. Though the role of TNF- $\alpha$ in TBI has not been fully elucidated, this response potentially indicates improved JM4 efficacy associated with more optimal inflammatory attenuation by slightly delayed initial therapy.

Though much optimism has surrounded the use of whole EPO and its derivatives in TBI, little is still known about possible mechanisms of action in neuroprotection. In a mouse model of EAE we found that EPO exhibits a strong immunomodulatory effect by inhibiting the proliferation of dendritic cell and antigen-specific T-cell populations while downregulating major histocompatibility complex-II and proinflammatory cytokine (IFN- $\gamma$, IL-2, IL-6, TNF- $\alpha$ ) expression. Wholemolecule EPO promoted the expansion of T regulatory cells, while also causing a striking decrease in peripheral $\mathrm{T}$ helper 17 cell levels [29]. All of these effects occurred in the lymph nodes and spleen (peripheral lymphoid system), as well as within the damaged target organ (spinal cord). This characteristic has also been retained in the JM4 molecule as we have demonstrated that JM4 suppresses IL-2, IL-5, IL-6, TNF- $\alpha$, and IFN- $\gamma$ while limiting peripheral mononuclear and dendritic cell populations in EAE-diseased animals [17]. In modulating multiple immune response cell types and their corresponding cytokines, EPO and its JM4 derivative transformed a highly uncontrolled autoimmune process into a more normalized condition while providing significant neuroprotective benefits in both clinical deficit and neuropathology.

Our small peptide EPO derivative JM4 was administered to acutely brain-injured mice. JM4 offers a therapeutic window of at least $9 \mathrm{~h}$ following injury. Results indicated remarkable reduction in neural cell death following TBI. JM4 shows promise as an effective tool in treating acute TBI and perhaps a broader range of acute neurological insults.

Acknowledgments We thank Kevin Pang, $\mathrm{PhD}$, for his help with animal studies and clinical scoring data analysis. We also thank William Banks, MD (Seattle, WA, USA), for his aid with the blood-brain barrier JM4 studies. This work was funded by the VA Office of Research and Development (IO1 RX000220-01; PD), the Segal Fund, and the NJ Brain Injury Commission (08-3208-BIR-E-1; PD).

\section{Compliance with ethical standards}

Required Author Form Disclosure forms provided by the authors are available with the online version of this article

\section{References}

1. Mammis A, McIntosh TK, Maniker AH. Erythropoietin as a neuroprotective agent in traumatic brain injury Review. Surg Neurol 2009;71(5):527-531.

2. Cherian L, Goodman JC, Robertson C. Neuroprotection with erythropoietin administration following controlled cortical impact injury in rats. J Pharmacol Exp Ther 2007;322(2):789-794. 
3. Mahmood A, Lu DY, Qu CS, et al. Treatment of traumatic brain injury in rats with erythropoietin and carbamylated erythropoietin. J Neurosurg 2007;107(2):392-397.

4. Siren AL, Fasshauer T, Bartels C, Ehrenreich H. Therapeutic Potential of erythropoietin and its structural or functional variants in the nervous system. Neurotherapeutics 2009;6(1):108-127.

5. Brines ML, Ghezzi P, Keenan S, et al. Erythropoietin crosses the blood-brain barrier to protect against experimental brain injury. Proc Natl Acad Sci U S A 2000;97(19):10526-10531.

6. Grasso G. Neuroprotective effect of recombinant human erythropoietin in experimental subarachnoid hemorrhage. J Neurosurg Sci 2001;45(1):7-14.

7. Adembri C, Massagrande A, Tani A, et al. Carbamylated erythropoietin is neuroprotective in an experimental model of traumatic brain injury. Crit Care Med 2008;36(3):975-978.

8. Erbayraktar S, Grasso G, Sfacteria A, et al. Asialoerythropoietin is a nonerythropoietic cytokine with broad neuroprotective activity in vivo. Proc Natl Acad Sci U S A 2003;100(11):6741-6746.

9. Wang L, Zhang ZG, Wang Y, Zhang RL, Chopp M. Treatment of stroke with erythropoietin enhances neurogenesis and angiogenesis and improves neurological function in rats. Stroke 2004;35(7): 1732-1737.

10. Wang Y, Zhang ZG, Rhodes K, et al. Post-ischemic treatment with erythropoietin or carbamylated erythropoietin reduces infarction and improves neurological outcome in a rat model of focal cerebral ischemia. Br J Pharmacol 2007;151(8):1377-1384.

11. Ehrenreich $\mathrm{H}$, Weissenborn $\mathrm{K}$, Prange $\mathrm{H}$, et al. Recombinant human erythropoietin in the treatment of acute ischemic stroke. Stroke 2009;40(12):E647-E656.

12. Corwin HL, Gettinger A, Fabian TC, et al. Efficacy and safety of epoetin alfa in critically ill patients. N Engl J Med 2007;357(10): 965-976.

13. Dicato M. Venous thromboembolic events and erythropoiesisstimulating agents: An update. Oncologist 2008;13:11-15.

14. Shelton SB, Pettigrew DB, Hermann AD, et al. A simple, efficient tool for assessment of mice after unilateral cortex injury. J Neurosci Methods 2008;168(2):431-442.

15. Robertson CS, Cherian L, Shah M. Neuroprotection with an erythropoietin mimetic peptide (pHBSP) in a model of mild traumatic brain injury complicated by hemorrhagic shock. J Neurotrauma 2012;29:1156-1166.

16. Brines M, Patel NS, Villa P, et al. Nonerythropoietic, tissueprotective peptides derived from the tertiary structure of erythropoietin. Proc Natl Acad Sci U S A 2008;105(31):10925-10930.

17. Yuan R, Wang B, Lu W, Maeda Y, Dowling P. A distinct region in EPO that induces immuno/inflammatory modulation and tissue protection. Neurotherapeutics 2015;12:850-861.

18. Brines M, Cerami A. Erythropoietin-mediated tissue protection: reducing collateral damage from the primary injury response. $\mathrm{J}$ Intern Med 2008;264(5):405-432.

19. Ahmet I, Tae HJ, Juhaszova M, et al. A Small nonerythropoietic helix B surface peptide based upon erythropoietin structure is cardioprotective against ischemic myocardial damage. Mol Med 2011;17(3-4):194-200.

20. Ueba H, Brines M, Yamin M, et al. Cardioprotection by a nonerythropoietic, tissue-protective peptide mimicking the $3 \mathrm{D}$ structure of erythropoietin. Proc Natl Acad Sci U S A 2010;107(32):14357-14362.

21. Chen H, Luo B, Yang X, et al. Therapeutic effects of nonerythropoietic erythropoietin analog ARA290 in experimental autoimmune encephalomyelitis rat. J Neuroimmunol 2014;268(12):64-70.

22. Vlodavsky E, Palzur E, Soustiel JF. Hyperbaric oxygen therapy reduces neuroinflammation and expression of matrix metalloproteinase-9 in the rat model of traumatic brain injury. Neuropathol Appl Neurobiol 2006;32(1):40-50.

23. Lewen A, Fujimura M, Sugawara T, Matz P, Copin JC, Chan PH. Oxidative stress-dependent release of mitochondrial cytochrome $\mathrm{c}$ after traumatic brain injury. J Cereb Blood Flow Metab 2001;21(8): 914-920.

24. Robertson CS, Hannay HJ, Yamal JM, et al. Effect of erythropoietin and transfusion threshold on neurological recovery after traumatic brain injury: a randomized clinical trial. JAMA 2014;312(1):36-47.

25. Imai N, Higuchi M, Kawamura A, et al. Physicochemical and biological characterization of asialoerythropoietin - suppressive effects of sialic acid in the expression of biological activity of human erythropoietin in vitro. Eur J Biochem 1990;194(2):457-462.

26. Doggrell SA. A neuroprotective derivative of erythropoietin that is not erythropoietic. Expert Opin Invest Drugs 2004;13(11):15171519.

27. Leist M, Ghezzi P, Grasso G, et al. Derivatives of erythropoietin that are tissue protective but not erythropoietic. Science 2004;305(5681):239-242.

28. Dalgard CL, Cole JT, Kean WS, et al. The cytokine temporal profile in rat cortex after controlled cortical impact. Front Mol Neurosci 2012;5:6.

29. Yuan RR, Maeda Y, Li WP, Lu W, Cook S, Dowling P Erythropoietin: a potent inducer of peripheral immuno/ inflammatory modulation in autoimmune EAE. PLOS ONE 2008;3(4):e1924.

30. Chen G, Shi JX, Hang CH, Me WY, Liu J, Liu XM. Inhibitory effect on cerebral inflammatory agents that accompany traumatic brain injury in a rat model: a potential neuroprotective mechanism of recombinant human erythropoietin (rhEPO). Neurosci Lett 2007;425(3):177-182.

31. Yatsiv I, Grigoriadis N, Simeonidou C, et al. Erythropoietin is neuroprotective, improves functional recovery, and reduces neuronal apoptosis and inflammation in a rodent model of experimental closed head injury. FASEB J 2005;19:1701-1703.

32. Wei X, Li Y, Sun X, et al. Erythropoietin protects against murine cerebral malaria through actions on host cellular immunity. Infect Immun 2014;82(1):165-173.

33. Stover JF, Schoning B, Beyer TF, Woiciechowsky C, Unterberg AW. Temporal profile of cerebrospinal fluid glutamate, interleukin-6, and tumor necrosis factor-alpha in relation to brain edema and contusion following controlled cortical impact injury in rats. Neurosci Lett 2000;288(1):25-28.

34. Fan L, Young PR, Barone FC, Feuerstein GZ, Smith DH, McIntosh TK. Experimental brain injury induces differential expression of tumor necrosis factor-alpha mRNA in the CNS. Mol Brain Res 1996;36(2):287-291. 